\title{
Compromiso gastrointestinal agudo en pacientes con dengue por serotipo 4. Comunicación de un caso y revisión de la literatura
}

\author{
Johan Marín, Stalin Vilcarromero, Brett M. Forshey, Juan C. Celis-Salinas, Cesar Ramal-Asayag, \\ Amy C. Morrison, Alberto Laguna-Torres, Martín Casapía y Eric S. Halsey
}

\section{Acute gastrointestinal involvement in dengue disease by serotype 4: a case report and literature review}

Dengue fever is the world's most important arboviral disease, presenting a wide clinical spectrum. We report for the first time in Peru, a case caused by dengue virus serotype 4 with significant gastrointestinal involvement (acute acalculous cholecystitis and acute hepatitis). In addition we carried out a review of the literature atypical presentation illustrating the importance of the characteristics of abdominal pain (right upper quadrant); presence of Murphy's sign, ultrasound, and liver enzymes levels, for appropriate diagnosis and clinical management.

Key words: Dengue, dengue virus serotype 4, acute acalculous cholecystitis, acute hepatitis.

Palabras clave: Dengue, dengue serotipo 4, colecistitis aguda alitiásica, hepatitis.

\section{Introducción}

$\mathrm{E}$ 1 dengue es la arbovirosis más frecuente en humanos y puede ser causado por cualquiera de los cuatro serotipos del virus del dengue (DENV-1, $-2,-3$ y -4$)$.

El virus del dengue pertenece a la familia Flaviridae y el género Flavivirus. Es causante de un amplio espectro clínico que va desde una enfermedad leve a moderada o dengue sin signos de alarma hasta una enfermedad grave o dengue grave ${ }^{1}$. La infección también ha sido asociada a presentaciones clínicas atípicas, poco frecuentes pero graves como la hepatitis aguda fulminante y la colecistitis aguda alitiásica (CAA) ${ }^{2}$.

En Perú, hasta el año 2009, las publicaciones sobre casos de dengue graves y/o dengue hemorrágico $(\mathrm{DH})$ son escasas, y corresponden a infecciones por DENV-3 $3^{3,4}$ pero ninguna refiere presentaciones atípicas o compromiso hepático. Sin embargo, sí se han descrito casos de dengue que desarrollaron colecistitis aguda alitiásica por ese mismo serotipo (Sihuincha, comunicación personal).

La hepatitis aguda ha sido comunicada en Sudaméri$\mathrm{ca}^{5-8}$ y Asia (Tailandia), donde se ha descrito desde una transaminemia leve a moderada hasta un daño hepático grave con casos fatales 9 . En contraste, la CAA definida por la presencia del signo de Murphy positivo y engrosamiento de la pared vesicular en la ultrasonografía, ha sido reportada con mayor frecuencia en el sudeste asiático ${ }^{10} \mathrm{y}$ en la región del Océano Indico ${ }^{2,11-17}$.

Presentamos el primer caso en el Perú, de un paciente infectado por el virus del dengue serotipo 4 , que desarrolló dengue con signos de alarma, colecistitis aguda alitiásica y hepatitis aguda. Además realizamos una revisión de la literatura científica sobre este tipo de presentación.

\section{Caso clínico}

Paciente de sexo femenino, de 22 años, residente y procedente de la ciudad de Iquitos, sin antecedentes mórbidos. Inició su enfermedad (día 1) con fiebre, mialgias, dolor óseo, cefalea y dolor retro-ocular. Ante la persistencia de estos síntomas los días 2 y 3 , la paciente consultó en un centro de salud donde se le diagnosticó un dengue sin signos de alarma, con rash, por lo que se le indicó paracetamol (500 mg tres veces al día) e hidratación oral. En el contexto del proyecto de investigación "Vigilancia y etiología de la enfermedad febril aguda en el Perú", a la paciente se le solicitó una muestra de sangre venosa para ser enviada al laboratorio del Naval Medical Research Unit Six (NAMRU-6) para realizar una reacción de polimerasa en cadena (RPC) para dengue y leptospirosis, IgM por método de ELISA y cultivo viral para otras arbovirosis. Al quinto día de enfermedad se agregó dolor abdominal moderado a intenso, tipo cólico, localizado en el epigastrio e hipocondrio derecho, asociado a náuseas y vómitos. En los días 6 y 7 los síntomas aumentaron en intensidad por lo que consultó nuevamente. Recibió hidratación y analgesia intravenosa; sin embargo, al no presentar mejoría se decidió su internación. Ingresó en mal estado general, quejumbrosa, con una temperatura de $37,6^{\circ} \mathrm{C}$ axilar, presión arterial $90 / 60 \mathrm{mmHg}$, frecuencia
Hospital Regional de Loreto, Iquitos, Perú (JM, JCC, CRA, MC). Naval Medical Research Unit Six (NAMRU-6), Iquitos, Perú (SV, BMF, ALT, ESH). University of California, Davis, Estados Unidos (ACM).

Estudio financiado por The United States Department of Defense Global Emerging Infections Systems Research Program, Work Unit No. 847705.82000.25GB.B0016. El proyecto "Vigilancia y etiología de las enfermedades febriles agudas en el Perú" fue aprobado por el Comité de Ética en Investigación del NMRC en Silver Spring, Maryland, E.U.A, por el Instituto Nacional de Salud y la Dirección General de Epidemiologia como un proyecto de vigilancia.

Los autores declaran no tener conflictos de interés.

Recibido: 20 de septiembre de 2012

Aceptado: 31 de mayo de 2013

Correspondencia a:

Stalin Vilcarromero stalinf@yahoo.com 
Figura 1. Ultrasonografía inicial. Se aprecia vesícula alitiásica con pared engrosada.

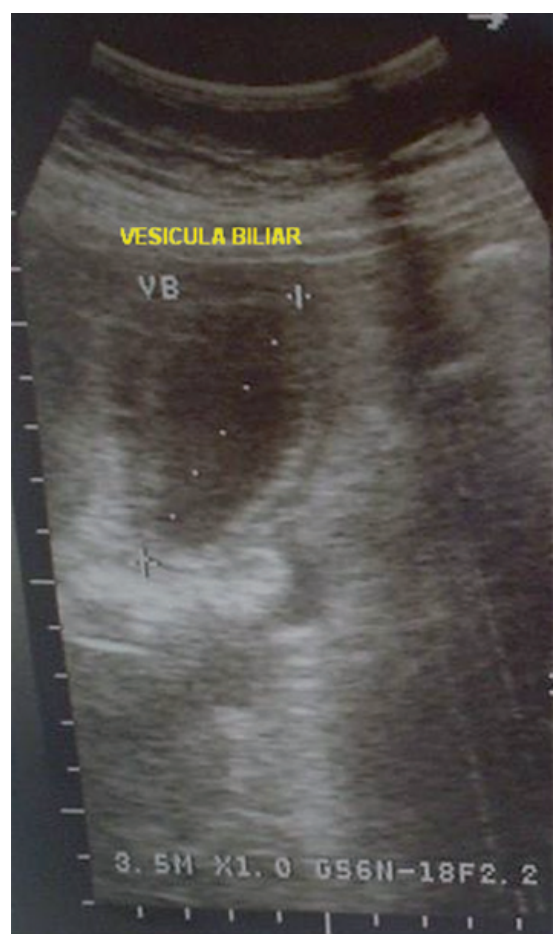

tratamiento de soporte hasta el día 12, cuando se decidió su alta médica por mejoría clínica parcial (disminución del dolor abdominal y ausencia de náuseas y vómitos) con diagnóstico de dengue y colecistitis alitiásica. La sintomatología disminuyó progresivamente y el día 25 se realizó un control de transaminasas ALT y AST que fueron de 18 y $25 \mathrm{U} / \mathrm{L}$, respectivamente.

El análisis de las muestras para descartar otras infecciones resultaron negativas: leptospirosis (RPC), rickettsiosis (IgG), hepatitis B (HBsAg) y otras arbovirosis como la encefalitis equina venezolana, mayaro, oropuche, fiebre amarilla y virus del grupo C (serología IgM y aislamiento viral). La gota gruesa para malaria también resultó negativa. La RPC para dengue resultó positiva para el virus dengue serotipo 4 .

\section{Discusión}

Iquitos es la ciudad más grande del noreste amazónico peruano, con una población de casi 370.000 habitantes ${ }^{18}$. Desde el año 1990, funciona un proyecto de vigilancia pasiva de arbovirosis, realizado por el Centro de Investigación de Enfermedades Tropicales de la Marina de los E.U.A (NAMRU-6), dentro del marco de un trabajo colaborativo con el Ministerio de Salud (Instituto Nacional de Salud y Dirección General de Epidemiología) y la Dirección Regional de Salud Loreto. Este proyecto incluye el estudio de pacientes con sindrome febril agudo (mayores de cinco años de edad, menos de siete días de fiebre y sin foco evidente) que acuden a alguno de los establecimientos de salud de esta ciudad ${ }^{19}$. Esta investigación ha permitido conocer que en Iquitos, en el período 2000-2009, los cuatro serotipos del dengue estuvieron presentes en el siguiente orden: DENV-1 (1990), DENV-2 (1995-2002), DENV-3 (2002-2008), DENV-4 (2008). Los casos más graves (DH grado III y IV) e incluso fatales fueron causados por el serotipo $3^{20}$. El DENV-4, a pesar de ser uno de los últimos en emerger, finalmente afectó gran parte de la población y logró desplazar completamente al DENV- $3^{19}$.

Es interesante comentar que cuando el DENV-4 apareció por primera vez en 2008, y empezó a dispersarse por toda la ciudad, se produjeron dos eventos importantes: la circulación, a menor escala, pero simultánea del DENV-3 y el incremento notable de personas febriles (casos probables y confirmados de dengue no grave). Estas condiciones eran favorables para esperar un incremento en los casos graves de dengue y/o DH; sin embargo, esto no se produjo ${ }^{21}$, concordando con la percepción actual de la comunidad médica local de que este serotipo era menos virulento. Un estudio realizado en Martinica (Caribe), donde hubo una epidemia de dengue por dos serotipos (2 y 4), demostró que el primero, asociado a infecciones secundarias, causó una mayor gravedad clínica ${ }^{22}$, hallazgo 
similar a lo encontrado en Colombia ${ }^{23}$. Otro estudio de dengue en Brasil, encontró una mayor gravedad con el serotipo $3^{24}$, incluso en infecciones primarias ${ }^{25}$. En la mayoría de las publicaciones, el serotipo 4 parece ser menos virulento; sin embargo, recientemente se ha publicado un caso fatal por DENV-4 ${ }^{26}$.

En este contexto, presentamos el caso de una mujer joven que inició su enfermedad con síntomas característicos de dengue (fase febril) y que en el $4^{\circ}$ día de enfermedad inició síntomas gastrointestinales (dolor abdominal agudo asociado a náuseas y vómitos) que aumentaron en intensidad y frecuencia, a pesar de la terapia de soporte establecida. Dada la evolución clínica, el equipo médico debió descartar otras causas de dolor abdominal.

El dolor abdominal durante la fase febril del dengue puede ser parte de la sintomatología general inespecífica pero generalmente, no es de aparición brusca, no es intenso y su frecuencia puede depender del serotipo involucrado. La infección con DENV-2 tiene una mayor frecuencia de este síntoma ${ }^{22,27}$ seguido por DENV-3 ${ }^{28}$. Según datos del proyecto de vigilancia, en 2.333 pacientes febriles, entre el año 2000 y 2009, en quienes se logró identificar el serotipo infectante, mediante aislamiento viral o RPC para dengue, sólo 875 presentaron dolor abdominal en la etapa aguda de la enfermedad, asociado en orden decreciente a DENV-4, DENV-3 y DENV-1 (Tabla 1). Los vómitos se asociaron con mayor frecuencia con DENV-3. Durante la fase crítica, el dolor abdominal puede representar un signo de alarma para dengue grave. Se caracteriza por ser un dolor intenso, localizado en el epigastrio y/o hipocondrio derecho y, puede o no, estar acompañado de otros signos de alarma (vómitos persistentes, postración extrema, etc.).

Según la última guía de la $\mathrm{OMS}^{1}$, se define dengue grave si se cumple una de las tres condiciones siguientes:

- Extravasación plasmática que origina hipotensión arterial (shock) y/o dificultad respiratoria.

- Hemorragia grave (criterio médico).

- Compromiso grave de algún órgano (hígado, sistema nervioso central, corazón y otros órganos).

El mayor número de pacientes con dengue grave presenta la primera condición, lo cual está relacionado con el mayor grado de permeabilidad vascular y consiguiente extravasación plasmática (ascitis, efusión pleural y/o edema pulmonar). Los exámenes de imagen demostraron en nuestro paciente, ascitis y efusión pleural, pero debido a la ausencia de dificultad respiratoria o shock no permitió considerarlo como un caso de dengue grave. Según la guía anterior de la OMS $(1997)^{29}$, recién el día 8 el paciente completó los cuatro criterios para dengue hemorrágico (DH) grado I, al resultar positiva la prueba del torniquete o lazo. Sin embargo, el dolor abdominal en el hipocondrio derecho, persistente e intenso, con signo de
Tabla 1. Pacientes con síntomas gastrointestinales. Vigilancia pasiva de pacientes febriles. Iquitos, Perú. 2000-2009

\begin{tabular}{|c|c|c|c|c|c|}
\hline \multirow{3}{*}{$\begin{array}{l}\text { Dengue } \\
\text { serotipos }\end{array}$} & \multirow{3}{*}{$\begin{array}{c}\text { n de } \\
\text { casos* }^{*}\end{array}$} & \multicolumn{4}{|c|}{ Número de casos con síntomas gastrointestinales } \\
\hline & & DA & $\mathbf{N}$ & v & $\mathrm{DA}+\mathrm{N}+\mathrm{V}$ \\
\hline & & n $\quad(\%)$ & n $\quad(\%)$ & n $(\%)$ & n $\quad(\%)$ \\
\hline DENV-1 & 58 & $15(25,9)$ & $31(53,4)$ & $10(17,2)$ & $4 \quad(6,9)$ \\
\hline DENV-3 & 1.659 & $604(36,4)$ & $1.143(68,9)$ & $576(34,7)$ & $280(16,9)$ \\
\hline DENV-4 & 610 & $254(41,6)$ & $403(66,1)$ & $157(25,7)$ & $97(15,9)$ \\
\hline Total general & 2.333 & $875(37,5)$ & $1.581(67,8)$ & $746(32,0)$ & $383(16,4)$ \\
\hline
\end{tabular}

Murphy positivo, obligó, desde un inicio, a considerar el diagnóstico de colecistitis aguda y en el contexto de una persona con dengue y sin antecedentes de litiasis vesicular, el considerar la CAA.

La CAA es una inflamación de la vesícula biliar sin evidencia de cálculo o barro. Se describe principalmente en pacientes críticos, en quienes los síntomas, como el dolor abdominal o signo de Murphy, están ausentes ${ }^{30}$. También puede presentarse secundariamente a algunas infecciones como fiebre tifoidea, leptospirosis, rickettsiosis, brucelosis, hepatitis A, dengue y otros ${ }^{2} \mathrm{y}$, en estos casos, existen síntomas asociados. En el dengue el diagnóstico se basa en el cuadro clínico y ultrasonográfico (dolor abdominal en el hipocondrio derecho, signo Murphy positivo, ausencia de litiasis vesicular y un engrosamiento de la pared vesicular mayor de $3 \mathrm{~mm}$ ). El engrosamiento vesicular es un hallazgo relativamente frecuente en el dengue, pero por sí sólo no constituye una $\mathrm{CAA}^{31,32}$.

No existen reportes de dengue complicados con CAA en nuestro país; sin embargo, en Brasil y, especialmente en Asia, existen varias publicaciones (Tabla 2). Al analizarlas, muchas no distinguen el paciente que presenta colecistitis al inicio, de aquel que desarrolla esta complicación durante el curso de la enfermedad. Sin embargo, existen coincidencias: todos presentaron dolor abdominal en el hipocondrio derecho, signo de Murphy positivo (clínicamente o con el transductor del ultrasonógrafo), engrosamiento de la pared vesicular con ausencia de litiasis. El tratamiento de elección fue médico y aquellos que fueron sometidos a cirugía o algún procedimiento quirúrgico, por sospecha de abdomen agudo, tuvieron una mayor estadía hospitalaria ${ }^{33}$. Algunos pacientes con colecistitis aguda por dengue presentaron signos peritoneales ${ }^{12,15,34}$. En casi todos los reportes no se precisó el serotipo involucrado.

Nuestra paciente tenía diagnóstico confirmado de dengue y CAA; sin embargo, la persistencia del dolor abdominal exigió descartar el compromiso de otros órganos intra-abdominales como hígado y páncreas. 


\begin{tabular}{|c|c|c|c|c|c|c|}
\hline \multirow[t]{2}{*}{ Autor } & \multirow[t]{2}{*}{ País } & \multirow{2}{*}{$\begin{array}{l}\text { Casos de } \\
\text { colecistitis/ } \\
\text { casos de } \\
\text { dengue }\end{array}$} & $\begin{array}{l}\text { Signos y síntomas } \\
\text { gastrointestinales }\end{array}$ & Transaminemia & $\begin{array}{l}\text { Estudio imagenológico } \\
\text { (Ultrasonografía) }\end{array}$ & Evolución \\
\hline & & & $\begin{array}{c}\text { Dolor abdominal (DA) } \\
\text { Dolor abdominal en el } \\
\text { hipocondrio derecho (DAHD) } \\
\text { Signo de Murphy (SM) }\end{array}$ & (AST, ALT) & $\begin{array}{l}\text { Engrosamiento de la pared } \\
\text { vesicular (EPV) } \\
\text { SM ultrasonográfico (SM-US) }\end{array}$ & $\begin{array}{c}\text { Buena (B) } \\
\text { Complicada (C) } \\
\text { Estadía hospitalaria (EH) }\end{array}$ \\
\hline $\begin{array}{l}\text { Wu KL, } \\
\text { et al }{ }^{11}\end{array}$ & Taiwan & 10/131 & $\begin{array}{l}\text { SM }(+)=100 \% \\
\text { Vómitos }=40 \%\end{array}$ & $\begin{array}{l}\mathrm{ALT}=96 \pm 48 \mathrm{U} / \mathrm{L} \\
\mathrm{AST}=186 \pm 122 \mathrm{U} / \mathrm{L}\end{array}$ & $\begin{array}{l}\text { EPV } \\
\text { Líquido pericolecístico SM-US (+) }\end{array}$ & $\begin{array}{l}\text { C: Los sometidos a cirugía tuvieron } \\
\text { mayor EH (18,16 días), } \\
\text { B: Resto tuvo } 4,1 \pm 1,6 \text { días de } \mathrm{EH}\end{array}$ \\
\hline $\begin{array}{l}\text { Sood A, } \\
\text { et al }{ }^{12}\end{array}$ & India & $1 / 1$ & $\begin{array}{l}\text { DAHD, rigidez abdominal, } \\
\text { vómitos, }\end{array}$ & $\begin{array}{l}\mathrm{ALT}=94 \mathrm{U} / \mathrm{L} \\
\mathrm{AST}=140 \mathrm{U} / \mathrm{L}\end{array}$ & $\begin{array}{l}\text { EPV }=6 \mathrm{~mm} \\
\text { Líquido pericolecístico SM-US (+) }\end{array}$ & B \\
\hline $\begin{array}{l}\text { Goh, BK, } \\
\text { et al'13 }\end{array}$ & Singapur & $1 / 1$ & DAHD, SM (+) & $\begin{array}{l}\mathrm{ALT}=215 \mathrm{U} / \mathrm{L} \\
\mathrm{AST}=418 \mathrm{U} / \mathrm{L}\end{array}$ & $\begin{array}{l}\text { Tomografía: EPV, } \\
\text { líquido pericolecístico }\end{array}$ & C: mayor EH \\
\hline $\begin{array}{l}\text { Sharma } \mathrm{N} \text {, } \\
\text { et a } \mathrm{l}^{14}\end{array}$ & India & $14 / 27$ & $\mathrm{DA}=100 \%$ & & $\begin{array}{l}E P V=5,2 \pm 1 \mathrm{~mm} \text {, estriamiento } \\
\text { de la pared vesicular, SM-US (+) }\end{array}$ & B: mayor EH (> 1,68 días) \\
\hline \multirow{3}{*}{$\begin{array}{l}\text { Jaufeerally } \\
F R \text {, et al }{ }^{15}\end{array}$} & \multirow[t]{3}{*}{ Singapur } & $2 / 2$ & & & & \\
\hline & & Caso 1 & $\begin{array}{l}\text { DAHD, Dolor epigástrico, } \\
\text { signo rebote }(+)\end{array}$ & $\mathrm{ALT}, \mathrm{AST}>6 \mathrm{v}$ & EPV & B \\
\hline & & Caso 2 & DAHD, signo rebote $(+)$ & ALT, AST > $10 \mathrm{v}$ & EPV & B \\
\hline $\begin{array}{l}\text { Joshipura VP, } \\
\text { et al }{ }^{16}\end{array}$ & India & $1 / 1$ & DAHD, SM (+) & $\begin{array}{l}\text { ALT }=89 \mathrm{U} / \mathrm{L} \\
\mathrm{AST}=108 \mathrm{U} / \mathrm{L}\end{array}$ & $\begin{array}{l}\text { EPV, ascitis, hepatomegalia, } \\
\text { esplenomegalia }\end{array}$ & B \\
\hline \multirow[t]{2}{*}{$\begin{array}{l}\text { De Souza } \sqcup, \\
\text { et al }\left.\right|^{34}\end{array}$} & \multirow[t]{2}{*}{ Brasil } & $\begin{array}{c}2 / 2 \\
\text { Caso 1: }\end{array}$ & $\begin{array}{l}\text { DAHD y epigastrio, SM (+), } \\
\text { náuseas, vómitos }\end{array}$ & $\begin{array}{l}\mathrm{ALT}=283 \mathrm{U} / \mathrm{L} \\
\mathrm{AST}=144 \mathrm{U} / \mathrm{L}\end{array}$ & EPV, líquido libre pélvico & $\mathrm{B}=12$ días $\mathrm{EH}$ \\
\hline & & Caso 2: & $\begin{array}{l}\text { DA, DAHD y epigastrio. SM } \\
(+)\end{array}$ & $\begin{array}{l}\mathrm{ALT}=30 \mathrm{U} / \mathrm{L} \\
\mathrm{AST}=42 \mathrm{U} / \mathrm{L}\end{array}$ & EPV, moderada ascitis & $\mathrm{B}=8$ días $\mathrm{EH}$ \\
\hline
\end{tabular}

Esto último se descartó con valores normales de amilasa sérica y una tomografía computarizada que no mostró daño pancreático.

El compromiso hepático se produce en casi todos los casos de dengue y se manifiesta por una elevación de las enzimas hepáticas o transaminasas (ALT y AST), a lo cual algunos han denominado "hepatitis reactiva". Se asume que es secundario a una respuesta inflamatoria del hospedero. Usualmente, este incremento de las transaminasas es menor a diez veces su valor normal y presenta sus valores máximos en la segunda semana ${ }^{35}$. Los valores de AST se elevan con una frecuencia y proporción mayor que las ALT, lo que podría explicarse debido al compromiso del virus en el sistema músculoesquelético $^{36}$. Esta diferencia puede ser de utilidad para distinguir el dengue de otras enfermedades febriles ${ }^{37}$, con excepción de la fiebre tifoidea ${ }^{38}$ y malaria ${ }^{39}$ las que podrían presentar el mismo patrón. Los valores de transaminasas mayores a diez veces el valor normal representan un signo de alarma para la enfermedad grave (dengue grave o DH) y hemorragia gastrointestinal ${ }^{40}$ pero también, un mayor riesgo de desarrollar insuficiencia hepática aguda y muerte sin necesidad de reunir otros criterios de gravedad o de $\mathrm{DH}^{41}$. Existe una asociación entre altos niveles de transaminasas e infección secundaria, independiente del serotipo presente, y además, existe una correlación entre los niveles de carga viral y de enzimas hepáticas ${ }^{22}$. Tanto la infección secundaria como la carga viral están asociadas a mayor gravedad. Esto es importante de considerar, ya que en nuestra región es infrecuente la solicitud de transaminasas en los pacientes con dengue, ya sea por una limitada capacidad logística de nuestros hospitales o por la creencia de que este tipo de complicación no es frecuente en nuestra región.

El mecanismo exacto de la patogénesis del compromiso hepático aún no está bien esclarecido; sin embargo, existen algunas evidencias de que podría deberse a: un efecto directo del virus, secundario a una respuesta inflamatoria del hospedero ${ }^{42}$, uso excesivo de paracetamol (dosis mayores de $4 \mathrm{~g} /$ día) o por efecto de alguna hepatopatía crónica concomitante.

Con respecto al efecto directo del virus, se ha encontrado la presencia de antígenos virales en el tejido hepático. Es probable que el virus tenga la capacidad de replicarse, tanto en los hepatocitos como en las células de Kupffer ${ }^{42}$. Cada serotipo podría estar asociado con diferentes grados de compromiso hepático ${ }^{43}$, tal es así que DENV 1, 2 y 3 han sido aislados en tejido hepático de pacientes fallecidos 


\begin{tabular}{|c|c|c|c|c|c|c|}
\hline \multirow[t]{2}{*}{ Autor } & País & $\begin{array}{c}n \text { de casos y } \\
\text { diagnóstico clínico } \\
\text { de dengue }\end{array}$ & $\begin{array}{l}\text { Signos y síntomas } \\
\text { gastrointestinales }\end{array}$ & Transaminemia & Ultrasonografía & Evolución \\
\hline & & $\begin{array}{c}\text { Dengue } \\
\text { hemorrágico (DH) } \\
\text { Dengue clásico (DC) }\end{array}$ & $\begin{array}{c}\text { Dolor abdominal (DA), DA } \\
\text { en el hipocondrio derecho } \\
\text { (DAHD), náuseas (N), vómitos } \\
(V) \text {, hepatomegalia }(H)\end{array}$ & $\begin{array}{c}\text { AST, ALT } \\
\text { (Valor promedio) }\end{array}$ & $\begin{array}{l}\text { Hepatomegalia }(\mathrm{H}) \text {, } \\
\text { Esplenomegalia }(\mathrm{E})\end{array}$ & $\begin{array}{c}\text { Buena (B) } \\
\text { Otra (O) }\end{array}$ \\
\hline $\begin{array}{l}\text { Del Valle } \\
\text { Díaz S, } \\
\text { et al }{ }^{5}\end{array}$ & Brasil & 37 casos con DH & $\begin{array}{c}\text { DAHD }=45,9 \% \\
H=83,8 \%\end{array}$ & $\begin{array}{l}\mathrm{ALT}=93,5 \mathrm{U} / \mathrm{L} \\
\mathrm{AST}=104 \mathrm{U} / \mathrm{L}\end{array}$ & $\begin{array}{c}\mathrm{H}=31 \text { casos asociados a: } \\
\text { edema peri-vesicular }(48,6 \%) \\
\text { líquido libre } \\
\text { intra-abdominal }(37,8 \%) \\
\mathrm{E}=24,3 \%\end{array}$ & B \\
\hline $\begin{array}{l}\text { Mourao MP, } \\
\text { et al }\end{array}$ & Brasil & 1 caso de $\mathrm{DH}$ & $\mathrm{DAHD}=100 \%, \mathrm{~V}, \mathrm{H}$ & $\begin{array}{l}\text { ALT }=2.129 \mathrm{U} / \mathrm{L} \\
\text { AST }=7.082 \mathrm{U} / \mathrm{L}\end{array}$ & No se menciona & B \\
\hline $\begin{array}{l}\text { Uehara PM, } \\
\text { et al }\end{array}$ & Brasil & 41 casos de $\mathrm{DH}$ & $\begin{array}{c}\mathrm{DA}=46 \%(19 / 41) \\
\mathrm{N} \text { y } \vee=90 \%(37 / 41)\end{array}$ & $\begin{aligned} \mathrm{ALT} & =89 \mathrm{U} / \mathrm{L} \\
\mathrm{AST} & =104 \mathrm{U} / \mathrm{L}\end{aligned}$ & No se menciona & B \\
\hline $\begin{array}{l}\text { Valero NC, } \\
\text { et al }{ }^{9}\end{array}$ & Venezuela & $\begin{array}{c}184 \text { casos } \\
\text { DC }=25 \% \\
\text { DH }=73,5 \%\end{array}$ & $\begin{array}{l}\text { 128/184 (46,8\%) pacientes } \\
\text { con afección hepática } \\
\text { (ictericia, H, DAHD) }\end{array}$ & $\begin{array}{c}112 / 184(60,8 \%) \text { casos de } \\
\text { dengue con transaminemia } \\
\text { ALT }=21,3 \pm 8 \text { y AST }=37,2\end{array}$ & No se menciona & $\begin{array}{l}\text { O: mayor } \\
\text { transaminemia en } \\
\text { dengue vs control }\end{array}$ \\
\hline $\begin{array}{l}\text { Parkash O, } \\
\text { et al| }{ }^{41}\end{array}$ & Pakistan & $\begin{array}{c}699 \text { casos } \\
D C=86 \%(605) \\
D H=13 \%(94)\end{array}$ & $\begin{array}{c}\mathrm{DA}=18,2 \%, \mathrm{DAHD}=7 \% \\
\mathrm{~N} \text { y } \mathrm{V}=48,6 \% \\
\text { hematemesis }=5,2 \% \\
\text { ictericia }=3,1 \%\end{array}$ & $\begin{array}{c}\text { Hepatitis-dengue (ALT): } \\
\text { leve-moderada: } \\
\text { (hasta } 10 \mathrm{v})=71 \%, \text { grave }=15 \% \\
\text { y normal }=14 \%\end{array}$ & $\begin{array}{l}\text { Colecistitis aguda } \\
\text { alitiásica }=3,9 \%\end{array}$ & $\begin{array}{l}\text { O: Mayor mortalidad } \\
\text { en el grupo de } \\
\text { hepatitis grave }\end{array}$ \\
\hline $\begin{array}{l}\text { De Souza ப, } \\
\text { et al }{ }^{49}\end{array}$ & Brasil & $\begin{array}{c}1.585 \text { casos } \\
\mathrm{DC}=91 \%(1.443) \\
\mathrm{DH}=9 \%(142)\end{array}$ & No se menciona & $\begin{array}{c}\text { ALT o AST: normal }=34,8 \%(551) \\
\text { leve }<3 v=44,5 \%(706) \\
\text { moderado } 3-10 v=16,9 \%(268) \\
\text { grave }>10 v=3,8 \%(60)\end{array}$ & No se menciona & $\begin{array}{l}\text { O: sólo } 4,2 \% \text { tuvo } \\
\text { hepatitis aguda } \\
\text { (no grave) }\end{array}$ \\
\hline
\end{tabular}

por insuficiencia hepática fulminante, lo que no ha ocurrido con el DENV-4 ${ }^{44-46}$. Un estudio en Bangkok, reportó un mayor compromiso hepático por los serotipos 3 y $4^{47}$, pero Kumaria, en la India, lo demostró con DENV-2 27.

Por otra parte, los hallazgos de Trung respecto a la evolución de las transaminasas y de otros marcadores de gravedad sugiere que la respuesta inmune del hospedero, sería el factor más importante en la patogénesis. Esto se observa en la coincidencia del pico máximo de las transaminasas en la fase crítica del dengue, donde se sabe que algunas complicaciones (trombocitopenia grave o shock) están determinadas por la respuesta inmune. Esto produce una activación de citoquinas, presencia de linfocitos $\mathrm{T}$ en tejidos y presencia de complejos inmunes ${ }^{48}$.

En relación al efecto del paracetamol, nuestra paciente aseguró haber tomado paracetamol a dosis de 1,5 g/día, lo que excluyó el efecto medicamentoso.

En cuanto a la co-infección con otra hepatitis viral, se descartó la hepatitis B, patología frecuente en nuestra región. En un estudio de 69 pacientes con hepatitis B estudiados por cursar con un episodio agudo de dengue, sólo se pudo demostrar un incremento de ALT sin repercusión en el grado de extravasación, hemorragia, trombocitopenia, shock o gravedad clínica de la hepatitis $\mathrm{B}^{35}$.
En el dengue pareciera existir alguna relación entre CAA y hepatitis grave, ya que muchas de las CAA presentan elevaciones leves a moderadas de las transaminasas, siendo infrecuente el compromiso hepático grave. Por su parte, la hepatitis grave en el dengue no es frecuente ${ }^{35}$, se presenta generalmente dentro de un cuadro de dengue clásico o dengue hemorrágico, y en ambos casos, la mortalidad ha sido elevada ${ }^{41}$. En dos de las series revisadas el dolor abdominal está presente en menos de $50 \%$ y la hepatomegalia tiene un rango muy variable, por lo que no es imprescindible para definir hepatitis por dengue (Tabla 3).

En resumen, se presenta el primer caso documentado en el Perú, de dengue causado por el serotipo 4 con compromiso gastrointestinal (colecistitis aguda alitiásica y hepatitis aguda moderada). Se debería considerar esta complicación en todo paciente con dengue que presenta dolor abdominal persistente en el hipocondrio derecho, asociado a náuseas y vómitos. Sin embargo, deben considerarse también otros diagnósticos diferenciales de abdomen agudo. El signo de Murphy y la ultrasonografía permiten el diagnóstico de colecistitis, mientras que la medición de las enzimas hepáticas (ALT y AST) establecen el grado de compromiso hepático. 
Agradecimientos: Juan López, Julio Canelo, Jorge Sibina y Jaime Salinas del Hospital Regional de Loreto. Gabriela Vásquez, Rubiela Rubio, Leslye Angulo y Nadia Montes de NAMRU 6 Iquitos. Christopher Cruz, Enrique Canales y Sonia Ampuero de NAMRU-6 Lima y Kanya C. Long de la Universidad de California en Davis.

\section{Resumen}

El dengue es la arbovirosis más importante del mundo y causa un amplio espectro clínico. Presentamos el primer caso de dengue causado por el serotipo 4 (DENV-4) en Perú con compromiso gastrointestinal (colecistitis aguda alitiásica y hepatitis aguda moderada). Se presenta una revisión de la literatura médica sobre este tipo de presentación, enfatizando la importancia y características del dolor abdominal (hipocondrio derecho), el signo de Murphy, los hallazgos ultrasonográficos y la medición de las enzimas hepáticas para establecer el diagnóstico y manejo adecuado.

\section{Referencias bibliográficas}

1.- World Health Organization. Dengue. Guidelines for diagnosis, treatment, prevention and control. Geneva: WHO; 2009.

2.- Gulati S, Maheshwari A. Atypical manifestations of dengue. Trop Med Int Health 2007; 12: 1087-95

3.- Ramal C, Orts C, Chauca H, Mestanza M. Dengue hemorrágico en el Hospital Regional de Loreto. Reporte de un caso. Rev Med Hered 2008; 19: 81-3.

4.- Nunura J, Benites C, Sihuincha M. Caso de dengue hemorrágico en Iquitos. Rev Peru Med Exp Salud Publica 2005; 22 (3): 229-32.

5.- Del Valle Díaz S, Piñera Martínez M, Guasch Saent-Félix F. Reactive hepatitis caused by the hemorrhagic dengue virus. Rev Cubana Med Trop 2001; 53: 28-31.

6.- Mourao M P, Lacerda M V, Bastos M de S, Albuquerque B C, Alecrim W D. Dengue hemorrhagic fever and acute hepatitis: a case report. Braz J Infect Dis 2004, 8: 461-4

7.- Uehara P M, da Cunha R V, Pereira G R, de Oliveira P A. Envolvimento hepático em pacientes com dengue hemorrágico: manifestação rara?. Rev Soc Bras Med Trop 2006; 39: 544-7.

8.- Méndez A, González G. Manifestaciones inusuales del dengue hemorrágico en niños. Biomédica 2006; 26: 61-70

9.- Valero N, Reyes I, Larreal I, Maldonado M. Aminotransferasas séricas en pacientes con dengue tipo 3. Rev Med Chile 2007; 135 : 1304-12.

10.- Poororawan $Y$, Hutagalung $Y$, Chongsrisawat $V$, Boudville I, Bock HL. Dengue virus infection: a major cause of acute hepatic failure in Thai children. Ann Trop Paediatr 2006; 26: 17-23.

11.- Wu K L, Changchien C S, Kuo C M, Chuah S K, Lu S N, Eng H L, et al. Dengue fever with acute acalculous cholecystitis. Am J Trop Med Hyg 2003; 68: 657-60.

12.- Sood A, Midha V, Sood N, Kaushal V. Acalculous cholecystitis as an atypical presentation of dengue fever. Am J Gastroenterol 2000; 95: 3316-7.
13.- Goh B K, Tan S G. Case of dengue virus infection presenting with acute acalculous cholecystitis. J Gastroenterol Hepatol 2006; 21: 923-4.

14.- Sharma N, Mahi S, Bhalla A, Singh, V, Varma S, Ratho R K. Dengue fever related acalculous cholecystitis in a North Indian tertiary care hospital. J Gastroenterol Hepatol 2006; 21: 664-7.

15.- Jaufeerally F R, Surrun S K, Chang P E. Acute acalculous cholecystitis in dengue hemorrhagic fever. Indian J Med Sci 2007; 61: 613-4.

16.- Joshipura V P, Soni H N, Patel N R, Haribhakti S P. Dengue fever presenting as acute acalculous cholecystitis. J Indian Med Assoc 2007; 105: 338-9.

17.- Karunatilake H, Vithiya K, Arasalingam A, Malavan R, Kumara D S. Acalculous cholecystitis and dengue fever. Ceylon Med J 2008; 53: 30 .

18.- Instituto Nacional de Estadística e Informática (INEI), 2008. Censos Nacionales 2007: XI de Población y VI de Vivienda, Sistema de Consulta de Resultados Censales, Cuadros Estadísticos. Accedido: julio de 2011 Disponible en: http://desa.inei.gob.pe/censos2007/ tabulados/

19.- Forshey B M, Morrison A C, Cruz C, Rocha C, Vilcarromero S, Guevara C, et al. Dengue virus serotype 4, northeastern Peru, 2008. Emerg Infect Dis 2009; 15: 1815-8

20.- Forshey B M, Guevara C, Laguna-Torres V A, Céspedes M, Vargas J, Gianella A, et al. Arboviral etiologies of acute febrile illnesses in Western South America, 2000-2007. PLoS Negl Trop Dis 2010; 4: e787.

21.- Dirección salud Loreto, Análisis de la Situación de Salud de la Región Loreto (ASIS-Loreto), 2009. http://www.diresaloreto.gob.pe/portal/ index.php/boletines-epidemiologicos (accedido en julio de 2011).

22.- Thomas L, Verlaeten O, Cabié A, Kaidomar S, Moravie V, Martial J, et al. Influence of the dengue serotype, previous dengue infection, and plasma viral load on clinical presentation and outcome during a dengue- 2 and dengue- 4 co-epidemic. Am J Trop Med Hyg 2008; 78: 990-8.
23.- Ocazionez R E, Gómez S Y, Cortés F M. Dengue hemorrhagic fever serotype and infection pattern in a Colombian endemic area. Rev Salud Publica 2007; 9: 262-74.

24.- Guilarde A O, Turchi M D, Siqueira J B Jr, Feres V C, Rocha B, Levi J E, et al. Dengue and dengue hemorrhagic fever among adults: clinical outcomes related to viremia, serotypes and antibody response. J Infect Dis 2008; 197: 817-24.

25.- Nisalak A, Endy T P, Nimmannitya S, Kalayanarooj S, Thisayakorn U, Scott R M, et al. Serotype-specific dengue virus circulation and dengue disease in Bangkok, Thailand from 1973 to 1999. Am J Trop Med Hyg 2003; 68: 191-202.

26.- Cecilia D, Kakade M B, Bhagat A B, Vallentyne J, Singh A, Patil J A, et al. Detection of dengue-4 virus in Pune, Western India after an absence of 30 years-its association with two severe cases. Virol J 2011; 8: 46.

27.- Kumaria R. Correlation of disease spectrum among four dengue serotypes: a five years hospital based study from India. Braz J Infect Dis 2010; 14: 141-6.

28.- Passos M N, Santos L M, Pereira M R, Casali C G, Fortes B de P, Ortiz Valencia L I, et al. Clinical differences observed in patients with dengue caused by different serotypes in the epidemic of $2001 / 2002$, occurred in Rio de Janeiro. Rev Soc Bras Med Trop 2004; 37 : 293-5.

29.- World Health Organization. Dengue haemorrhagic fever: diagnosis, treatment, prevention and control. 2nd ed. Geneva: WHO; 1997.

30.- Indar A A, Beckingham I J. Acute cholecystitis. Br Med J 2002; 325: 639-43.

31.- Venkata Sai P M, Dev B, Krishnan R. Role of ultrasound in dengue fever. Br J Radiol 2005; 78: 416-8

32.- Oliveira G A, Machado R C, Horvat J V, Gomes L E, Guerra L R, Vandesteen L, et al. Transient reticular gallbladder wall thickening in severe dengue fever: a reliable sign of plasma leakage. Pediatr Radiol 2010; 40: 720-4.

33.- Khor B S, Liu J W, Lee I K, Yang K D. Dengue hemorrhagic fever patients with acute 
abdomen: clinical experience of 14 cases. Am J Trop Med Hyg 2006; 74: 901-4.

34.- de Souza L J, Zagne S M O, Araujo P G, Zagne L O, Maciel N D S, Araujo F N. Acalculous cholecystitis in dengue. Case reports. Rev Bras Clin Med 2009; 7: 56-9.

35.- Trung D T, Thao le T T, Hien T T, Hung N T, Vinh N N, Hien P T, et al. Liver involvement associated with dengue infection in adults in Vietnam. Am J Trop Med Hyg 2010; 83: 774-80.

36.- Salgado D M, Eltit J M, Mansfield K, Panqueba C, Castro D, Vega M R, et al. Heart and skeletal muscle are targets of dengue virus infection. Pediatr Infect Dis J 2010; 29: 238-42.

37.- Kalayanarooj S, Vaughn D W, Nimmannitya S, Green S, Suntayakorn S, Kunentrasai N, et al. Early clinical and laboratory indicators of acute dengue illness. J Infect Dis 1997; 176: 313-21.

38.- Morgenstern R, Hayes P C. The liver in typhoid fever: always affected, not just a complication. Am J Gastroenterol 1991; 86: 1235-9.

39.- Patwari A, Aneja S, Berry A M, Ghosh S. Hepatic dysfunction in childhood malaria. Arch Dis Child 1979; 54: 139-41.

40.- Wichmann O, Hongsiriwon S,
Bowonwatanuwong C, Chotivanich $\mathrm{K}$, Sukthana Y, Pukrittayakamee S. Risk factors and clinical features associated with severe dengue infection in adults and children during the 2001 epidemic in Chonburi, Thailand. Trop Med Int Health 2004; 9: 1022-29.

41.- Parkash O, Almas A, Jafri SM, Hamid S, Akhtar J, Alishah H. Severity of acute hepatitis and its outcome in patients with dengue fever in a tertiary care hospital Karachi, Pakistan (South Asia). BMC Gastroenterol 2010; 10: 43.

42.- Seneviratne S L, Malavige G N, de Silva H J. Pathogenesis of liver involvement during dengue viral infections. Trans R Soc Trop Med Hyg 2006; 100: 608-14.

43.- Vásquez-Pichardo M, Rosales-Jiménez C, Rojas-Espinosa O, López-Martínez I, Moreno-Altamirano M M B. Liver damage dependent on the serotype of dengue virus? A study in Mexico. Dengue Bull 2006; 30: 114-20.

44.- Sumarmo, Wulur H, Jahja E, Gubler D J, Suharyono W, Sorensen K. Clinical observations on virologically confirmed fatal dengue infections in
Jakarta, Indonesia. Bull World Health Org 1983; 61 (4): 693-701.

45.- Miagostovich M P, dos Santos F B, de Araújo E S, Dias J, Schatzmayr H G, Nogueira R M. Diagnosis of dengue by using reverse transcriptase-polymerase chain reaction. Mem Inst Oswaldo Cruz 1997; 92: 595-9.

46.- Basílio-de-Olivera C A, Aguiar G R, Baldanza M S, Barth O M, Eyer-Silva W A, Paes M V. Pathologic study of a fatal case of dengue-3 virus infection in Rio de Janeiro, Brazil. Braz J Infect Dis 2005; 9: 341-7.

47.- Kalayanarooj S, Nimmannitya S. Clinical and laboratory presentations of dengue with different serotypes. WHO Dengue Bull 2000; 24: 53-9.

48.- Mathew A, Rothman A L. Understanding the contribution of cellular immunity to dengue disease pathogenesis. Immunol Rev 2008; 225 : 300-13.

49.- Souza L J, Alves J G, Nogueira R M, Gicovate Neto C, Bastos D A, Siqueira E W, et al. Aminotransferase changes and acute hepatitis in patients with dengue fever: analysis of 1,585 cases. Braz J Infect Dis 2004, 8: $156-63$ 